\title{
Preface: DGAA Special Issue on Numerical Methods for Dynamic Games
}

\author{
Herbert Dawid $^{1}$ • Maurizio Falcone ${ }^{2}$ (ID
}

Published online: 13 May 2017

(C) Springer Science+Business Media New York 2017

\section{Introduction}

The theory of dynamic games has progressed very much in the last decades, and these models are now used in many areas which span from economics to engineering applications, from decision models in social sciences to computer science. Just to quote two typical examples, the allocation of investments in dynamic industry models or the optimal policy design in (multicountry) macroeconomic settings can be formulated as a dynamic game, the optimization of traffic flow on a network (which could be a road network or an Internet network) can also be formulated as a dynamic game. These new formulations have to be made effective via a quantitative solution of dynamic games models because realistic models in these fields are in general analytically intractable, clearly their treatment requires the use of numerical methods to obtain reliable approximate optimal strategies for the players. Another important issue is that these solutions should also be implemented in a reasonable amount of time in order to be really useful (just think at the regulation of informations flows on the Internet).

This is the main motivation to develop new algorithms and approximation schemes which could help in their numerical solution and to analyze their efficiency and complexity. It is also important to remind that the numerical solution of game problems is a rather difficult task, even for simple models. These problems often involve nonlinear terms and the space dimension is usually rather high either because of the number of players or because of the complexity of the state space. For example, for pursuit-evasion games when we have two players the space state is doubled unless one can use reduced coordinates expressing the position of one player in a reference system attached to the second player. This will imply to solve a problem in $\mathbb{R}^{2 d}$ if every player lives in $\mathbb{R}^{d}$. For other types of games when the

Maurizio Falcone

falcone@mat.uniroma1.it

Herbert Dawid

hdawid@wiwi.uni-bielefeld.de

1 Department of Business Administration and Economics, Bielefeld University, Bielefeld, Germany

2 Dipartimento di Matematica, Università degli Studi "La Sapienza”, Rome, Italy 
number of players increases the situation is even more complicated and one must rely on some aggregated variables. This is the case for mean-field games problems where the number of players is so huge that it becomes impossible to deal directly with every player. For $N$ players one gets a system of $N$ elliptic partial differential equations that provides a Nash equilibrium in feedback form for $N$-person stochastic differential games, and it can be proved that in the limit for $N$ going to infinity one gets a system of two PDEs, where a classical Hamilton-JacobiBellman equation is coupled with a Kolmogorov-Fokker-Planck equation for the density of the players. Numerical approaches to determine equilibria of dynamic games typically rely on dynamic programming with time discretization (e.g., [2], or [1]), collocation schemes (e.g., [5]) or the application of standard approaches, like finite elements or finite differences, for solving the associated Hamilton-Jacobi-Bellman equations [3,4].

The present issue contains both theoretical contributions, developing new numerical schemes for the computation of equilibria in certain classes of dynamic games, and applied papers, in which numerical schemes are employed to study equilibrium behavior in different concrete problems characterized by dynamic strategic interaction.

In the paper "Completely Mixed Strategies for Generalized Bimatrix and Switching Controller Stochastic Games" Dubey, Neogy and Ghorui consider switching controller stochastic games, i.e., bimatrix games with state-dependent payoff matrices, for which the transition rates between the states depend in each state only on the action of one of the two players. They formulate a (vertical) linear complementarity problem for characterizing equilibria of such games and use this approach to derive conditions under which switching controller stochastic games have completely mixed optimal stationary strategies. Furthermore, the vertical linear complementarity problem formulation is used to derive a numerical algorithm to calculate optimal completely mixed strategies with a substantially lower computational burden than full enumeration.

Also the contribution "Numerical Analysis of Markov-Perfect Equilibria with Multiple Stable Steady States: A Duopoly Application with Innovative Firms" by Dawid, Keoula and Kort deals with a dynamic game involving stochastic transitions between discrete states of the game, which in their framework are referred to as modes of the game to distinguish them from the continuously evolving state variables. Dawid et al. study Markov-Perfect Equilibria (MPE) of a multi-mode differential game describing the interaction between two competing incumbent firms investing in Research and Development (R\&D) in order to develop a new product to be introduced to the market. Innovation times are stochastic and the arrival rate of each firm's innovation depends on its R\&D investment and a public knowledge stock. Formally, each innovation corresponds to a mode transition in the game. A Chebychev polynomial-based collocation algorithm is developed to calculate MPEs of this game, where a special challenge lies in the fact that equilibrium strategies are not continuous everywhere on the two-dimensional state space and the state dynamics induced by MPE strategies has multiple coexisting stable steady states. Applying collocation methods to such games requires to derive conditions under which pasting together of profiles, which satisfy corresponding Hamilton-Jacobi-Bellman equations on parts of the state space, generates an MPE profile on the entire considered state space.

Wrcaczek, Kaplan, Caulkins, Seidl and Feichtinger in their paper "Differential Terror Queue Games" consider the dynamic strategic interaction between the government and a terrorist organization under different informational assumptions. They formulate a 2-player differential game, in which the total number of terror plots and the number of detected terror plots are the state variables. They compare open-loop equilibria, in which both players cannot react to changes in the states, with an asymmetric information case, in which each player can only react to changes in one of the two states, and with a closed-loop equilibrium in 
which all strategies are functions of both states and time. Relying on numerical solutions of the systems of state- and costate-dynamics in different scenarios they show as a main finding that the number of terror plots developed in the asymmetric information scenario is substantially larger than in both the open-loop and the closed-loop scenario.

The paper by Botkin, Turova, Diepolder, Bittner and Holzapfel "Aircraft Control During Cruise Flight in Windshear Conditions: Viability Approach," deals with a classical problem of aircraft control. They develop the analysis and approximation of aircraft control capabilities during the cruise phase in the presence of windshears. The study uses a point-mass aircraft model describing flight in a vertical plane. The problem is formulated as a differential game against wind disturbances where the first player (the autopilot) controls the angle of attack and the power setting, whereas the second player (the wind) produces dangerous gusts. The state variables of the model are subject to constraints expressing aircraft safety conditions. Namely, the altitude, path inclination, and velocity are constrained. Viability theory is used to find the so-called viability kernel, the maximal subset of the state constraint where the aircraft trajectories can remain arbitrary long if the first player uses an appropriate feedback control, and the second player generates any admissible disturbances.

The interesting survey by Kumkov, Patsko and Le Menec "Zero-Sum Pursuit-Evasion Differential Games with Many Objects: Survey of Publications" gives an updated overview of several contributions in the field of group pursuit games. If a pursuit game with many persons can be formalized in the framework of zero-sum differential games, then general methods can be applied to solve it. Several difficulties arise connected with very high dimension of the phase vector when there are too many objects. In order to solve this problem, special formulations and methods have been elaborated for conflict interaction of groups of objects and they are presented in this article.

The paper "A numerical algorithm to calculate the unique feedback Nash equilibrium in a large scalar LQ differential game" by Engwerda develops numerical algorithms for the characterization of feedback Nash equilibria of differential games. In particular, this paper considers different types of linear quadratic $N$-player games with a single state variable, distinguishing between games where players are interested in steering the state toward zero or making it as large as possible. Conditions for existence and uniqueness of feedback Nash equilibria in such games are provided and numerical algorithms for different scenarios are developed in order to determine efficiently existence and uniqueness of equilibria in (asymmetric) games with a large number of players. Furthermore, in case of unique equilibria the developed algorithms also compute the equilibrium strategy profiles.

In the paper by Gomes, Almulla and Ferreira "Two numerical approaches to stationary mean-field games" the authors propose numerical methods for stationary mean-field games (MFG) and investigate two classes of algorithms. The first one is a gradient-flow method based on the variational characterization of certain MFG. The second exploits monotonicity properties of MFG. The methods are illustrated by various examples including one-dimensional periodic MFG, congestion problems and higher-dimensional models.

Finally, the paper by Carlini, Festa, Silva and Wolfram "A Semi-Lagrangian scheme for a modified version of the Hughes model for pedestrian flow" deals with a numerical method for Mean Filed Models. In particular, they present a Semi-Lagrangian scheme for a regularized version of the Hughes model for pedestrian flow. Hughes originally proposed a coupled nonlinear PDE system describing the evolution of a large pedestrian group trying to exit a domain as fast as possible. The original model corresponds to a system of a conservation law for the pedestrian density coupled with an eikonal equation to determine the weighted distance to the exit. Here, they consider that model in presence of small diffusion and discuss the numerical analysis of the proposed Semi-Lagrangian scheme presenting various experiments. 
In conclusion, we wish to thank Georges Zaccour, Editor-in-Chief of Dynamic Games and Applications, for proposing a special issue on numerical methods, for inviting us to serve as guest editors of this journal and for his continuous support during the preparation of the volume. We also would also like to thank the authors who have contributed to this volume submitting their work and all the reviewers for their input on submitted papers. We hope that this special issue will inspire new contributions in this area.

\section{References}

1. Doraszelski U, Judd K (2012) Avoiding the curse of dimensionality in dynamic stochastic games. Quant Econ 3(2012):53-93

2. Falcone M (2006) Numerical methods for differential game based on partial differential equations. Int Game Theory Rev 8:231-272

3. Falcone M, Ferretti R (2014) Semi-Lagrangian approximation schemes for linear and Hamilton-Jacobi equations. SIAM

4. Jensen M, Smears I (2013) On the convergence of finite element methods for Hamilton-Jacobi-Bellman equations. SIAM J Numer Anal 51(1):137-162

5. Vedenov DV, Miranda MJ (2001) Numerical solution of dynamic oligopoly games with capital investment. Econ Theory 18:237-261 